\title{
Diferencias individuales y burnout en médicos oncólogos venezolanos
}

\section{Individual Differences and Burnout in Venezuelan Oncologists}

Recibido: enero 27 de 2011 | Revisado: julio 27 de 2011 | Aceptado: febrero 10 de 2012

\author{
BLANCA TORRES * \\ Hospital Oncológico Luis Razetti, Caracas, Venezuela \\ LETICIA GUARINO \\ Universidad Simón Bolívar, Caracas, Venezuela
}

SICI: 1657-9267(201303)12:1<95:DIBMOV>2.0.TX;2-F

Para citar este artículo: Torres, B. \& Guarino, L. (2013). Diferencias individuales y burnout en médicos oncólogos venezolanos. Universitas Psychologica, 12(1), 95-104.

\footnotetext{
Hospital Oncológico Luis Razetti. Calle Real de Cotiza. San José Cotiza, Caracas, Venezuela. E-mail: btorres@hotmail.com
}

\section{RES UMEN}

El presente estudio explora la influencia de ciertas diferencias individuales como la sensibilidad emocional, los estilos de afrontamiento y los estilos de comunicación sobre el burnout de médicos oncólogos venezolanos. Participaron en el estudio 93 médicos oncólogos de diversos hospitales oncológicos públicos de Caracas (Venezuela), quienes completaron los cuestionarios que evaluaban las variables mencionadas. Los resultados indicaron niveles medios de burnout en los médicos estudiados, una mayor sensibilidad emocional positiva, adopción de funcionales estilos de afrontamiento y estilos de comunicación más centrados en sí mismos. Por su parte, el análisis de regresión reveló que una mayor sensibilidad egocéntrica negativa y una menor sensibilidad interpersonal positiva determinan mayores niveles de burnout en los oncólogos.

Palabras clave autores

diferencias individuales, oncólogos, burnout.

Palabras clave descriptores

Psicología Organizacional, Psicología de la Salud, invesigación cuantitativa

\section{A B S T R A C T}

The present study explores the influence of certain individual differences such as the emotional sensitivity, the coping styles and the communication styles over the burnout of Venezuelan oncologists. 93 oncologists participated in the study from different public oncological hospitals from CaracasVenezuela, who completed the questionnaires evaluating the mentioned variables. Results indicated medium levels of burnout for the physicians, a higher positive emotional sensitivity, the use of functional coping styles and communication styles more self centered. On the other side, the regression analysis revealed that a higher negative egocentric sensitivity and a lower positive interpersonal sensitivity determine higher levels of burnout in the oncologists.

Key words authors

Individual differences, oncologists, burnout.

Key words plus

Work Psychology, Health Psychology, Quantitative Research. 


\section{Introducción}

Es una realidad mostrada en numerosos estudios, que el estrés experimentado en profesiones relacionadas con la salud es más alto que en el resto de las profesiones (Marrero \& Grau, 2005). Concretamente, el área médica es uno de los ámbitos donde los profesionales se ven sometidos a constante tensión emocional en la lucha por recuperar la salud del paciente.

En los últimos años, sobre todo a partir de la década de los ochenta, uno de los términos usados para describir el estrés en profesionales de la salud ha sido el Síndrome del Burnout. El término burnout fue introducido por Freudenberger (1974) para denotar al conjunto de síntomas físicos y signos conductuales que manifiesta el individuo al estrés laboral crónico.

Actualmente, se reconoce la definición establecida por Maslach (1982) como un síndrome de fatiga emocional, despersonalización y de un logro personal reducido, que puede ocurrir entre los individuos que trabajan directamente con pacientes. Es decir, como un síndrome de estrés crónico propio de aquellas profesiones de servicios que se caracterizan por una atención intensa y prolongada con personas que están en una situación de necesidad o dependencia.

Los profesionales de la salud, especialmente los médicos, se ven sometidos diariamente a presiones laborales que pueden originar niveles considerables de estrés y burnout, en detrimento de la calidad de atención al paciente y de su propia salud. En este sentido, hay evidencia que señala que existen algunas especialidades dentro del campo de la medicina que predisponen a los médicos a experimentar mayores niveles de estrés. Según Mingote, Moreno y Gálvez (2004), entre las unidades médicas que reportan alta vulnerabilidad al estrés laboral se encuentran aquellas clasificadas de atención primaria, servicios de salud mental, medicina interna, cardiología, unidades de enfermedades infecciosas, unidades de hemofilia, unidades de oncología y médicos residentes.

En el ámbito oncológico, además de la carga laboral propia de las labores asistenciales, se agre- gan la carga emocional de lidiar con el sufrimiento del paciente, la muerte y los conflictos propios del oncólogo. Como lo reportan muchas investigaciones, existen niveles altos de estrés y burnout en el personal que labora en el área oncológica (Arigoni, Mermillod, Sappino \& Waltz, 2006; Corti, Buscemi \& Font, 2006; Fallowfield, Jenkins, Catt, Langridge \& Cox, 2006; Price, Butow, Grivas, Webb \& Loos, 2006). Los médicos oncólogos constituyen, dentro de la medicina, una población vulnerable al estrés laboral, con su consecuente deterioro de la salud física y mental, así como también la disminución de la calidad productiva, en detrimento de los pacientes.

Existen diferencias individuales relacionadas con los niveles de estrés y burnout experimentados por los oncólogos. Una de las variables que se debe tomar en cuenta es el afrontamiento que utilizan los especialistas para manejar las situaciones estresantes. Existe evidencia que señala que los estilos de afrontamiento emocionales están asociados a la aparición de síntomas físicos y psicológicos, y el afrontamiento por evitación acentúa los efectos negativos del estrés sobre la salud; mientras que el afrontamiento racional y por desapego emocional actúan como variables moderadoras protectoras de los síntomas físicos (Aldana, 2010; Guarino, 2009; Herrera, 2006; Lousinha, 2009; Sojo, 2006).

La sensibilidad emocional es otra variable que puede estar asociada al nivel de burnout vivenciado por los oncólogos. Según Guarino (2005), el constructo define la alta disposición de los individuos a experimentar intensas emociones negativas frente a las demandas ambientales, así como la habilidad para reaccionar ante las emociones expresadas por otros. Esta variable ha sido estudiada en médicos de áreas críticas mostrando su relación con el estrés laboral y la aparición de síntomas físicos en estos profesionales (Bethelmy, 2006). Sin embargo, no se ha profundizado la investigación en el área oncológica, lo que será el objetivo de este estudio.

Otra de las variables que se debe tomar en cuenta en el estudio del burnout experimentado por los oncólogos, es el estilo de comunicación utilizado por el médico en la relación terapéutica con 
el paciente. Los hallazgos de las investigaciones en el área apuntan a un nivel mayor de satisfacción, bienestar e identificación tanto de pacientes como del mismo médico con un estilo de comunicación centrado en el paciente, ya que le permite al oncólogo brindar información de manera efectiva, a la vez que responder a sus propias necesidades emocionales y las del paciente (Dowsett et al., 2000; Jenkins \& Fallowfield, 2002; López, Pastor $\&$ Aubalat, 2006).

Los niveles de estrés y burnout experimentados por los oncólogos pueden generarle a largo plazo numerosos problemas de salud física y mental, así como la inadecuada adaptación de estos individuos a su entorno laboral, familiar y social, en detrimento a su vez de la atención y la salud del paciente. Se hace necesario entonces, promover el estudio del síndrome de burnout en estos profesionales para lograr una cabal comprensión del fenómeno y las variables involucradas en el mismo, con el objetivo último de prevenir y controlar su aparición a futuro.

Esta investigación pretende estudiar la capacidad predictiva de algunas diferencias individuales de los médicos oncólogos como son los estilos de afrontamiento, la sensibilidad emocional y los estilos de comunicación, sobre los niveles de burnout que pueden cursar estos profesionales de la salud en Venezuela.

El estudio representa un avance fundamental en la investigación del proceso del estrés y más en concreto del Síndrome de Burnout en el área oncológica, ya que permite conocer sus características en el contexto venezolano en el que ha sido poco investigado, y consecuentemente arrojar luces en cuanto al comportamiento de esta variable y los factores que podrían determinarla. A su vez, permitiría generar aportes en cuanto a la personalidad del oncólogo en relación con las variables que pueden servir de protectoras para un óptimo desempeño de su trabajo. De igual forma, los resultados de la investigación pueden derivar en programas de prevención y tratamiento para el manejo del estrés en este grupo de profesionales, así como también en habilidades de comunicación más efectivas con el paciente.

\section{Método}

\section{Participantes}

La muestra estuvo constituida por 93 médicos oncólogos que laboran en diversas instituciones públicas (hospitales oncológicos) del área metropolitana de Caracas-Venezuela, 59 \% hombres y 41 \% mujeres y con edades comprendidas entre los 29 y los 79 años (edad media $=42$ años).

\section{Instrumentos}

\section{Escala de Sensibilidad Emocional (ESE)}

La escala mide la sensibilidad emocional en sus tres dimensiones, a saber: positiva, negativa y distanciamiento emocional. En la presente investigación se utilizó la versión en español de Guarino y Roger (2005) compuesta por 45 ítems. La mencionada escala permite obtener un puntaje acerca de la reactividad emocional de las personas, ubicándolas en una de las dimensiones según sus características. La consistencia interna de la escala en la población hispanoparlante se considera satisfactoria, siendo $\alpha=0.824$ para el factor de sensibilidad egocéntrica negativa (SEN), $\alpha=0.742$ para el distanciamiento emocional (DE) y $\alpha=0.71$ para la sensibilidad interpersonal positiva (SIP). Las respuestas están reunidas en una escala de puntuación sumada tipo Likert, con opciones de respuesta de verdadero o falso para cada uno de los ítems. Las personas que contestan verdadero a los ítems, reflejan la presencia de las características propias de cada uno de los componentes descritos.

\section{Cuestionario de Estilos de Afrontamiento (CSQ)}

Para la medición de la variable estilos de afrontamiento, se utilizó la adaptación al español del Cuestionario de Estilos de Afrontamiento (CSQ) de Roger, Jarvis y Najarian (1993), desarrollada por Guarino, Sojo y Bethelmy (2007), la cual mide el afrontamiento en sus cuatro dimensiones, a saber: emocional, racional, evitación y el desapego emocional, aunque esta última dimensión es una subescala 
de la dimensión emocional. El instrumento está constituido por 40 ítems: el factor emocional está compuesto por 12 ítems, el racional se integra de 10 ítems, el de desapego se compone de 13 ítems y el de evitación consta de 5 ítems. Los índices de confiabilidad de las dimensiones de la escala son $\alpha=$ 0.78 para afrontamiento emocional, $\alpha=0.65$ para afrontamiento por evitación, $\alpha=0.73$ para desapego emocional y $\alpha=0.76$ para afrontamiento racional.

En este cuestionario se les pide a los participantes que ubiquen en una escala de frecuencia (siempre, frecuentemente, algunas veces o nunca) la forma en que típicamente reaccionan frente a determinadas situaciones. La corrección del inventario se realiza sumando los valores que en la escala de frecuencia los participantes asignaron en los ítems que corresponden a cada dimensión de la variable por separado. Los datos se interpretan en función de los descriptivos obtenidos.

\section{Maslach Burnout Inventory (MBI)}

Creada por Maslach et al. (1986), se utilizó la versión en español que contiene 22 ítems distribuidos en tres factores: agotamiento emocional (AE), despersonalización (D) y falta de realización personal en el trabajo (FRP). La escala de agotamiento emocional está compuesta por 9 ítems que describen sentimientos de estar abrumado y agotado emocionalmente por el trabajo; la escala de despersonalización está constituida por 5 ítems que se refieren a una respuesta impersonal y una falta de sentimientos hacia los sujetos objeto de la atención y la escala de falta de realización personal en el trabajo está compuesta por 8 ítems que describen competencia y realización exitosa en el trabajo hacia los demás.

En la subescala de despersonalización, puntuaciones $<6$ indican un nivel bajo de esta dimensión; entre 6 y 9 , un nivel medio y $\geq 10$, un nivel alto. En la dimensión falta de realización personal, un puntaje $\geq 40$ indica un nivel bajo en esta dimensión; entre 39 y 34 un nivel medio y $<34$, un nivel alto. En la subescala cansancio emocional puntuaciones $<19$ indican un nivel bajo de la dimensión; entre 19 y 26, un nivel medio y $\geq 27$, un nivel alto. En cuanto al puntaje total de la escala, puntuaciones $\leq 58$ indican un nivel bajo de burnout total; entre 58 y 76 , un nivel medio y $\geq 77$ un nivel alto.

Cuestionario de Estilos de

Comunicación del médico (ECM)

Para la medición de la variable estilos de comunicación, se utilizó el Cuestionario de estilos de Comunicación creado para los fines de esta investigación, que consta de dos dimensiones independientes, una centrada en el médico y otra centrada en el paciente. El instrumento está compuesto por 28 ítems. La dimensión centrada en el médico consta de 12 ítems y presenta una confiabilidad de $\alpha=0.761$. La dimensión centrada en el paciente está formada por 16 ítems, con una confiabilidad $\alpha=0.86$. La confiabilidad de la escala total fue de $\alpha=0.83$. Altos puntajes en cada una de las dimensiones, reflejan la presencia de la variable.

\section{Procedimiento}

Una vez que se tenían los instrumentos para medir las variables, se tramitaron los permisos en los centros oncológicos de Caracas (Padre Machado y Luis Razetti, respectivamente) para tener acceso a los oncólogos, cuya participación sería voluntaria. Se aplicaron los instrumentos organizados en forma de cuadernillo único y se recogió la información necesaria, aplicando los cuestionarios a los especialistas en su propio sitio de trabajo.

Con los datos obtenidos en la fase de recolección, se creó la base de datos a partir de la cual se realizaron los análisis estadísticos necesarios, utilizando el paquete estadístico SPSS versión 17.0 en español.

\section{Resultados}

Inicialmente se analizaron los datos descriptivos de las variables evaluadas para conocer su comportamiento en la población. La Tabla 1 muestra los valores para el burnout total y sus dimensiones.

Los datos indican un nivel moderado de burnout en la muestra de oncólogos estudiada. La variable 
TABLA 1

Estadísticos descriptivos del burnout total y sus dimensiones

\begin{tabular}{ccccccc}
\hline & M & DE & As & K & Rango Observ. & Rango Posible \\
\hline Burnout Total & 60.71 & 14.72 & 0.29 & -0.46 & $34-101$ & $0-132$ \\
\hline FRP & 38.4 & 6.14 & Dimensiones \\
AE & 16.4 & 11.26 & 0.87 & 0.51 & $20-48$ & $0-48$ \\
D & 1.07 & 0.32 & 1.11 & -0.49 & $2-48$ & $0-54$ \\
\hline
\end{tabular}

Nota. FRP: Falta de realización personal. AE: Agotamiento emocional. D: despersonalización.

Fuente: elaboración propia.

TABLA 2

Estadísticos descriptivos de la variable estilos de afrontamiento

\begin{tabular}{ccccccc}
\hline Dimensiones & M & DE & As & K & Rango Observ. & Rango Posible \\
\hline RAC & 22.11 & 3.93 & -0.46 & 0.34 & $12-30$ & $0-33$ \\
DES & 13.98 & 4.21 & 1.11 & 1.21 & $7-26$ & $0-39$ \\
EMO & 8.81 & 3.93 & 1.04 & 0.87 & $2-20$ & $0-33$ \\
EVI & 4.77 & 2.88 & 0.9 & 0.62 & $1-15$ & $0-15$ \\
\hline
\end{tabular}

Nota. RAC: Afrontamiento racional. DES: Afrontamiento por desapego emocional. EMO: Afrontamiento emocional. EVI: Afrontamiento por evitación.

Fuente: elaboración propia.

TABLA 3

Estadísticos descriptivos de la variable sensibilidad emocional

\begin{tabular}{ccccccc}
\hline Dimensiones & $\mathrm{M}$ & $\mathrm{DE}$ & $\mathrm{As}$ & $\mathrm{K}$ & Rango Observ. & Rango Posible \\
\hline SIP & 8.62 & 2.24 & -0.45 & -0.108 & $3-12$ & $0-13$ \\
SEN & 6.76 & 3.47 & 0.59 & 0.66 & $0-17$ & $0-22$ \\
DE & 3.78 & 2.14 & -0.51 & -0.68 & $0-9$ & $0-10$ \\
\hline
\end{tabular}

Nota. SIP: Sensibilidad interpersonal positiva. SEN: Sensibilidad egocéntrica negativa. DE: Distanciamiento emocional. Fuente: elaboración propia.

se observa poco dispersa, los datos se concentran más hacia la derecha de la media y ligeramente platicúrtica y el coeficiente Kolgorov-Smirnov sugiere una distribución normal para esta variable.

Analizando por separado las dimensiones de la variable burnout, puede observarse un puntaje más alto en la dimensión FRP (38.4) tomando en cuenta el puntaje observado de 48 como máximo y el mínimo 20. Los datos se agrupan en torno a la media con una ligera inclinación hacia la derecha de la misma, la cual no se distribuye normalmente. El AE reportó una puntuación promedio de 16.4 (puntajes observados entre 2 y 48). Las medidas de dispersión sugieren que los valores distan li- geramente hacia la derecha del valor central, aunque se distribuye normalmente. Por su parte, el puntaje promedio para la $\mathrm{D}$ fue de 1.07 , en un rango observado de 0 a 20, distribuyéndose los valores de forma más heterogénea hacia la derecha de la media, siendo una distribución desviada de la normal.

En síntesis, puede decirse que los médicos oncólogos del estudio presentan un nivel medio en cuanto a la dimensión FRP y un nivel bajo en relación con $\mathrm{AE}$ y $\mathrm{D}$, respectivamente. En relación con el nivel de burnout global, puede considerarse que los médicos presentan un nivel moderado del síndrome de burnout. 
La Tabla 2 muestra los datos descriptivos de la variable afrontamiento al estrés, en sus cuatro dimensiones.

El RAC es sin duda el más utilizado por los oncólogos, con una puntuación de 22.11, después aparece el estilo por DES con un puntaje de 13.98. Por otro lado, puede verse como el afrontamiento por EVI y el EMO son menos utilizados por los médicos de la muestra para manejar el estrés, lo que sugiere en general, un adecuado afrontamiento al estrés por parte de estos galenos.

La Tabla 3 muestra los datos descriptivos para la variable sensibilidad emocional, en sus tres dimensiones.

En la muestra de oncólogos, la SIP fue la dimensión con más alto puntaje, esto indica que los médicos son capaces de ser empáticos, reconocer las emociones de los otros y manejarlas de forma adecuada. La SEN es también una característica de personalidad que se encuentra presente en la muestra, aunque con menor fuerza, es decir que los oncólogos en menor medida centran sus emociones en sí mismos y se les dificulta el contacto emocional con los demás. El DE es la tercera dimensión que se presenta, dando cuenta de que los médicos también pueden eludir las emociones fuertes o perturbadoras de los otros, para evitar ser afectados por estas.
Finalmente, la Tabla 4 muestra los datos descriptivos de la variable estilos de comunicación en sus dos dimensiones.

En función de los puntajes ponderados, puede decirse que los oncólogos tienden a utilizar más el ECM en su dinámica de atención al paciente, lo que coincide con lo que se observa tradicionalmente en la relación médico-paciente, a pesar de no ser el estilo más idóneo en esta especialidad oncológica debido a las características de atención que ameritan los pacientes con cáncer.

Una vez obtenidos los resultados descriptivos de las variables, las puntuaciones de las mismas fueron utilizadas para calcular una ecuación de regresión, con el objeto de determinar el valor predictivo de los estilos de afrontamiento, la sensibilidad emocional y los estilos de comunicación en la explicación del burnout en los médicos oncólogos. La Tabla 5 muestra los resultados.

Como puede apreciarse, el burnout es explicado en primer lugar por la SEN solamente, la correlación obtenida es moderadamente alta (0.602) y explica el $35.5 \%$ de la varianza total, siendo esta una relación significativa $(F=49.95$; $p=0.001$ ), lo que implica que a altos puntajes en SEN corresponden altos puntajes en burnout.

TABLA 4

Estadísticos descriptivos de la variable estilos de comunicación

\begin{tabular}{ccccccc}
\hline Dimensiones & M & DE & As & K & Rango Observ. & Rango Posible \\
\hline ECP & 32.43 & 5.91 & 0.15 & -0.55 & $21-45$ & $0-48$ \\
ECM & 28.49 & 4.30 & -0.51 & -0.74 & $18-34$ & $0-36$ \\
\hline
\end{tabular}

Nota. ECP: Estilo centrado en el paciente. ECM: Estilo centrado en el médico.

Fuente: elaboración propia.

TABLA 5

Regresión entre variables independientes y burnout total

\begin{tabular}{cccccccc}
\hline & \multicolumn{7}{c}{ Variable dependiente: burnout } \\
\hline Modelo Variable & $\beta$ & $t$ & $p$ & $R$ & $\mathbf{R}^{2}$ & $\boldsymbol{F}$ & Sig. \\
\hline 1 SEN & 0.602 & 7.068 & 0.001 & 0.602 & 0.355 & 49.95 & 0.001 \\
2 SEN & 0.690 & 8.018 & 0.001 & 0.653 & 0.413 & 32.263 & 0.001 \\
SIP & -0.267 & -3.107 & 0.003 & & & & \\
\hline
\end{tabular}

Nota. SIP: sensibilidad interpersonal positiva. SEN: sensibilidad egocéntrica negativa.

Fuente: elaboración propia. 
El segundo modelo de regresión para predecir el burnout, incorpora además de la SEN a la variable SIP, la cual aumenta la varianza explicada en $41.3 \%$, con una correlación moderada entre las variables $(-0.267)$ que es negativa y significativa, tal como lo demuestra el valor de la $F=32.263$; $p=0.001$ ).

Los valores del índice de tolerancia para las variables $(\mathrm{SEN}=1 ; \mathrm{SIN}=0.891$ ) habla también de la independencia de las variables independientes y refuerza el modelo. El resto de las variables independientes quedaron excluidas del modelo de regresión. Este resultado sugiere que aquellos médicos que tengan alta SEN y baja SIP, son quienes experimentan mayores niveles de burnout total, independientemente de sus estilos de afrontamiento al estrés y de sus estilos de comunicación, al contrario de lo que se esperaba.

\section{Discusión}

El presente estudio se propuso determinar la capacidad predictiva de algunas diferencias individuales como la sensibilidad emocional, los estilos de afrontamiento y los estilos de comunicación sobre el nivel de burnout de médicos oncólogos de la ciudad de Caracas. Una revisión de los resultados descriptivos de las variables evidencia que el burnout muestra un nivel moderado del síndrome en la muestra de oncólogos estudiada. Este hallazgo coincide con investigaciones reportadas en el área, donde se encontraron niveles moderados en las muestras estudiadas como las de Marrero y Grau (2005), Aranda et al. (2006), Bernaldo y Labrador (2007) y Flores (2009).

Los oncólogos de los hospitales de Caracas estudiados experimentan un nivel medio de burnout, aunque se observaron diferencias en sus dimensiones. Específicamente, el AE y la D se presentaron en un nivel bajo, mientras que la FRP se presentó en un nivel medio. Estos hallazgos coinciden parcialmente con las investigaciones en el área como las de Arigoni et al. (2006), Corti et al. (2006) y Favara et al. (2008) que reportan niveles importantes de burnout en las profesiones asistenciales y concretamente en los médicos oncólogos. En este sentido, los médicos oncólogos están afectados medianamente por el burnout, sobre todo en lo referente a la percepción de que su trabajo no vale la pena, que institucionalmente es imposible cambiar las cosas, y que la mejora personal e institucional no merecen esfuerzo.

Tales niveles moderados de burnout se explican por las características intrínsecas de las instituciones hospitalarias donde laboran los galenos, en las cuales se enfrentan día a día con numerosas dificultades a la hora de desempeñar sus funciones, como el hecho de no poseer insumos básicos; la carencia de personal especializado que los obliga a trabajar más por el mismo salario, que ya es insuficiente para cubrir sus necesidades; realizar labores asistenciales, de formación y administrativas conjuntamente; equipos dañados, poco actualizados o, en el peor de los casos, inexistentes; poca estimulación organizacional y gubernamental para la investigación.

En relación con los estilos de comunicación, los resultados indican que la dimensión más utilizada en la muestra de oncólogos es la centrada en el médico. Los médicos se caracterizan por utilizar una comunicación donde el profesional se focaliza en la tarea, tiene un alto control sobre las conductas y muestra poca empatía con el paciente.

Esto apoya el modelo médico tradicional, en el cual el especialista ejerce el rol principal en la interacción, dejando al paciente un rol secundario y pasivo, quien solo ejecuta las instrucciones del médico. Estos hallazgos nos revelan una práctica común en nuestro país en cuanto a la relación médico-paciente, donde el médico da pocas oportunidades para que el paciente se involucre en la toma de decisiones de su propio proceso de enfermedad, lo que genera la mayoría de las veces ansiedad y poca satisfacción con la relación, tanto de parte del médico como del paciente.

Esto lo confirman investigaciones como la de Dowsett et al. (2000) y Shilling, Jenkins y Fallowfield (2003), quienes encontraron altos niveles de satisfacción de los pacientes cuyos médicos utilizan estilos de comunicación centrados en el paciente. También, Maguirre y Pitceathly (2002) señalan que 
cuando el médico logra identificar y entender los problemas importantes de sus pacientes, es capaz de generar mayor satisfacción con la atención recibida y alcanzar una mayor adherencia al tratamiento; finalmente, el médico incrementa su bienestar por los resultados adecuados en el manejo del paciente. Igualmente, Ruiz, Rodríguez, Perula y Prado (2002) encontraron una alta asociación entre la satisfacción del médico con su trabajo y el proceso de comunicación con el paciente.

Con respecto a los estilos de afrontamiento, el estilo racional fue el más utilizado por los médicos oncólogos del estudio, quienes se caracterizan por buscar todas las alternativas posibles para la solución real de las desavenencias generadas en un entorno estresante. Es decir, los médicos están en la búsqueda constante y dinámica de soluciones plausibles a los problemas que se puedan presentar en la dinámica de su trabajo y evitar, de esta manera, que los afecte o les genere tensión.

En segundo lugar, los médicos utilizan el desapego emocional como estrategia de afrontamiento ante las situaciones estresantes, separan de una manera adaptativa sus emociones de las de los demás, no dejando que las emociones y situaciones ajenas perturben de manera negativa el equilibrio interno personal y emocional. Los médicos que adoptan esta estrategia de afrontamiento son capaces de no involucrarse emocionalmente con sus pacientes, ni con sus familiares, a la vez que visualizan las experiencias estresantes en general desde una perspectiva objetiva.

Los estilos anteriores se muestran como deseables y adaptativos en el funcionamiento normal del médico en su trabajo y sirven como protectores en cuanto a su bienestar físico y psicológico. Este resultado, apoya el modelo de Roger et al. (1993), el cual expone que tanto la estrategia racional como la de desapego emocional constituyen los dos estilos de afrontamiento más adaptativos al estrés. Asimismo, estos resultados coinciden con los hallazgos reportados en el área por otros investigadores. Por ejemplo, Bethelmy (2006) encontró que los médicos de diversas disciplinas utilizaban los estilos de afrontamiento racional y por desapego emocional, y que estos resultaron ser moderadores de la relación entre el control decisional (componente del estrés laboral) y la manifestación de síntomas físicos.

Finalmente, en lo que se refiere a la sensibilidad emocional, la sensibilidad interpersonal positiva es la característica de personalidad que más se destaca en la muestra de oncólogos del presente estudio. Según Guarino (2004), esta característica describe la tendencia de ciertos individuos a orientarse emocionalmente hacia los otros, mostrando una gran habilidad para reconocer sus emociones, así como también manifestar preocupación y compasión por aquellos que sufren o atraviesan circunstancias difíciles.

Según esta evidencia, los oncólogos son capaces de ser empáticos con sus pacientes, entenderlos en su padecimiento y en sus necesidades y por tanto están más dispuestos a brindarles ayuda efectiva y oportuna. Este hallazgo apoya lo encontrado por Bethelmy (2006) en una muestra de médicos de áreas críticas de hospitales de Caracas, quienes también puntuaron alto en la sensibilidad interpersonal positiva sobre las otras dimensiones de la sensibilidad emocional, como son la sensibilidad egocéntrica negativa y el distanciamiento emocional.

Con respecto al análisis de regresión y la capacidad predictiva de las diferencias individuales, la sensibilidad emocional resultó ser la variable de personalidad que mejor explica la presencia de estrés en los médicos oncólogos. Específicamente, la sensibilidad egocéntrica negativa es la dimensión que mejor explica el nivel de burnout experimentado por los oncólogos, seguida por la dimensión sensibilidad interpersonal positiva, lo que sugiere que los oncólogos experimentan burnout de manera significativa cuando presentan altos niveles de sensibilidad egocéntrica negativa y poca sensibilidad interpersonal positiva.

Vale decir, los médicos con tendencia a reaccionar negativamente con emociones de aprehensión, rabia, desesperanza, vulnerabilidad y autocrítica ante las demandas ambientales y situaciones estresantes, por un lado, y que, por otro lado, no se orientan hacia los demás, ni muestran habilidad para reconocer sus emociones o manifestar compasión por su sufrimiento, son los que experimentan mayores niveles de estrés y, en este caso, del Síndrome de Burnout. 
Este hallazgo, aunque no en el área del burnout sino de los síntomas autorreportados, coincide con lo encontrado por otros autores que han estudiado la sensibilidad emocional, por ejemplo Guarino (2004), Herrera (2006), Bethelmy (2006) y Lousinha (2009), quienes reportaron que altos niveles de sensibilidad egocéntrica negativa predicen significativamente más reporte de síntomas físicos y psicológicos que otras diferencias individuales.

Se hace patente, entonces, la condición de riesgo que constituye la sensibilidad egocéntrica negativa en personas que están sometidas a situaciones difíciles y de alta demanda emocional en su trabajo, las profesiones asistenciales y en general en toda la población. La sensibilidad egocéntrica negativa pareciera ser una característica de personalidad que predispone de manera directa al estrés, con todas las consecuencias físicas y psicológicas que ello implica. Los estilos de afrontamiento y los estilos de comunicación no parecen tener una influencia directa sobre el burnout reportado por los médicos.

Este resultado sugiere la necesidad de desarrollar programas de intervención dirigidos a los médicos en general, y a los oncólogos en particular, para controlar o reducir su sensibilidad egocéntrica negativa y desarrollar mayor sensibilidad interpersonal positiva a la hora de desarrollar su labor asistencial con los pacientes, con el fin último de reducir sus probabilidades de experimentar altos niveles de burnout.

Sin embargo, debe enfatizarse que los resultados de este estudio revelaron solo niveles medios de burnout en la muestra de oncólogos, a diferencia de lo esperado, sugiriendo que estos galenos están parcialmente protegidos de los efectos devastadores de este síndrome. Tal protección se supone puede devenir de los adaptativos estilos de afrontamiento al estrés que utilizan estos especialistas (más afrontamiento racional y de desapego emocional), los cuales les permiten manejar de manera más adecuada las demandas del día a día del proceso hospitalario. Asimismo, pareciera estar beneficiando a la salud de los galenos el mostrar una baja sensibilidad egocéntrica negativa, así como una alta sensibilidad positiva, lo cual redunda en un mayor bienestar físico y psicológico.

\section{Referencias}

Aldana, G. (2010). Sobrecarga, afrontamiento y salud en cuidadoras de pacientes con demencia tipo Alzheimer. Trabajo de grado presentado para optar al grado de Magíster en Psicología, Universidad Simón Bolívar, Caracas, Venezuela.

Aranda, C., Pando, M., Torres, T., Salazar, J. \& Aldrete, M. (2006). Síndrome de burnout y manifestaciones clínicas en los médicos familiares que laboran en una institución de salud para los trabajadores del Estado. Psicología y Salud, 16(1), 15-21.

Arigoni, F., Mermillod, B., Sappino, A. \& Waltz, P. (2006, octubre). Emotional profile of doctors working in oncology: A Swiss French survey. Trabajo presentado en el 8th World Congress of PsychoOncology, Bolonia/Venecia, Italia.

Bernaldo, M. \& Labrador, J. (2007). Evaluación del estrés laboral y burnout en los servicios de urgencia extrahospitalaria. International Journal of Clinical and Health Psychology, 7(2), 323-335.

Bethelmy, L. (2006). Estrés laboral, diferencias individuales y salud en médicos de hospitales públicos de Caracas. Trabajo de grado presentado para optar al grado de Magíster en Psicología, Universidad Simón Bolívar, Caracas, Venezuela.

Corti, V., Buscemi, V. \& Font, A. (2006, octubre). Burnout Syndrome in oncology staff in Barcelona: Interpersonal relationship problems and intervention suggestions. Trabajo presentado en el 8th World Congress of Psycho-Oncology, Bolonia/ Venecia, Italia.

Dowsett, S., Saul, J., Butow, P., Dunn, S., Boyer, M., Findlow, R., et al. (2000). Communication styles in the cancer consultation: Preferences for a patientcentred approach. Psycho-Oncology, 9(2), 147-156.

Fallowfield, L., Jenkins, V., Catt, S., Langridge, C. \& Cox, A. (2006). Communication skills, burnout and the oncology team [Abstract]. Psycho-Oncology, 15(S2), 1-478.

Flores, P. (2009). Estrategias de afrontamiento y justicia organizacional sobre burnout en profesionales de salud mental. Trabajo de grado presentado para optar al grado de Magíster en Psicología, Universidad Simón Bolívar, Caracas, Venezuela. 
Freudenberger, H. (1974). Staff burn-out. Journal of Social Issues, 30(1), 159-166.

Guarino, L. (2004). Sensibilidad emocional: una nueva medida de labilidad emocional y su rol moderador en la relación estrés-enfermedad. Trabajo de ascenso para optar a la categoría de Profesor Asociado, Universidad Simón Bolívar, Caracas, Venezuela.

Guarino, L. (2005). Sensibilidad emocional: su rol moderador en la relación estrés-enfermedad. Psicología Conductual, 13(2), 217-230.

Guarino, L. (2009). Validación del modelo de sensibilidad emocional. Propuesta de un modelo de mediación moderada. Trabajo de ascenso para optar a la categoría de Profesor Titular, Universidad Simón Bolívar, Caracas, Venezuela.

Guarino, L. \& Roger, D. (2005). Construcción y validación de la Escala de Sensibilidad Emocional (ESE). Un nuevo enfoque para medir neuroticismo. Psicothema, 17(3), 465-470.

Guarino, L., Sojo, V. \& Bethelmy, L. (2007). Adaptación y validación preliminar de la versión hispana del Cuestionario de Estilos de Afrontamiento (Coping Style Questionnaire, CSQ). Psicología Conductual, 15(2), 173-189.

Herrera, V. (2006). Sensibilidad emocional y afrontamiento como moderadoras de la relación estrés-salud en cadetes. Trabajo de grado presentado para optar al grado de Magíster en Psicología, Universidad Simón Bolívar, Caracas, Venezuela.

Jenkins, V. \& Fallowfield, L. (2002). Can communication skills training alter physicians' beliefs and behaviour in clinics. Journal of Clinical Oncology, 20(3), 765-769.

López, S., Pastor, M. \& Aubalat, C. (2006). Comunicación en la consulta de oncología. Punto de Vista, mayo-junio, 10-14.

Lousinha, A. (2009). Diferencias individuales como moderadoras de la relación estrés-salud en docentes universitarios. Trabajo de grado presentado para optar al grado de Magíster en Psicología, Universidad Simón Bolívar, Caracas, Venezuela.

Maguirre, P. \& Pitceathly, C. (2002). Key communication skills and how to acquire them. BMJ, 325(7366), 697-700.

Marrero, M. \& Grau, J. (2005). Síndrome de Burnout en médicos que trabajan en unidades de cuidados intensivos neonatales. Psicología y Salud, 15(1), 25-32.

Maslach, C. (1982). Understanding burnout: Definitional issues in analyzing a complex phenomenon. En W. S. Paine (Ed.), Job stress and burnout (pp. 29-40). Beverly Hill: Sage.

Mingote, J., Moreno, B. \& Gálvez, M. (2004). Desgaste profesional y salud de los profesionales médicos: revisión y propuestas de prevención. Medicina Clínica, 123(7), 265-270.

Price, M., Butow, P., Grivas, D., Webb, P. \& Loos, C. (2006). Psychological distress, social support and unmet needs in ovarian cancer caregivers: How caregiver needs differ from patient needs [Abtract]. Psycho-Oncology, 15(S2), S1-S478.

Roger, D., Jarvis, G. \& Najarian, B. (1993). Detachment and coping: The construction and validation of a new scale for measuring coping strategies. Personality and Individual Differences, 15(6), 619-626.

Ruiz, M., Rodríguez, J., Perula, T. \& Prado, A. (2002). Evolución del perfil comunicacional de los médicos residentes de medicina de familia. Atención Primaria, 29(3), 132-134.

Shilling, V., Jenkins, V. \& Fallowfield, L. (2003). Factors affecting patient and clinician satisfaction with the clinical consultation: Can communication skills training for clinicians improve satisfaction? Psycho-Oncology, 12(6), 599-611.

Sojo, V. (2006). Evaluación de factores psicosociales relacionados con la salud global de un grupo de desempleados venezolanos. Trabajo de grado presentado para optar al grado de Magíster en Psicología, Universidad Simón Bolívar, Caracas, Venezuela. 